Language Shift in the United States 
Contributions to the Sociology of Language

$$
34
$$

Joshua A. Fishman

Editor

MOUTON PUBLISHERS - BERLIN · NEW YORK · AMSTERDAM 


\section{Language Shift in the United States}

Calvin Veltman 
Library of Congress Cataloging in Publication Data

Veltman, Calvin.

Language shift in the United States.

(Contributions to the sociology of language ; 34)

1. English language - United States - Social aspects.

2. English language - Acquisition. 3. Children -

United States - Language. I. Title. II. Series.

PE2808.V44 $1983 \quad 401^{\prime} .9 \quad 83-13272$

(C) Copyright 1983 by Walter de Gruyter \& Co., Berlin. All rights reserved, including those of translation into foreign languages. No part of this book may be reproduced in any form - by photoprint, microfilm, or any other means - nor transmitted nor translated into a machine language without written permission from the publisher. Typesetting: Grestun Graphics, Abingdon - Printing: Druckerei Hildebrand, Berlin. - Binding: Lüderitz \& Bauer Buchgewerbe GmbH, Berlin. - Cover design: K. Lothar Hildebrand, Berlin. 